\title{
Depression screening in cancer patients: A narrative review
}

\author{
Gulfama Abid, Vahe Kehyayan, Jessie Johnson* \\ Faculty of Nursing, University of Calgary Qatar, Qatar
}

Received: January 5, 2018

Accepted: February 26, $2018 \quad$ Online Published: June 7, 2018

DOI: $10.5430 /$ jnep.v8n11p11

URL: https://doi.org/10.5430/jnep.v8n11p11

\begin{abstract}
Depression is a significant and most common psychological symptom in cancer patients that causes a high risk of emotional and somatic consequences leading to poor quality of life. Despite the high prevalence of depression in cancer patients, the lack of screening and under-diagnosis of depression continues to be common. Numerous studies have shown that depression is a substantial complication in cancer patients that may lead to a variety of psychological and physical (somatic) symptoms. Presently, there are no depression screening practices at the Hamad Medical Corporation's (HMC) National Center for Cancer Care and Research (NCCCR), which is the principle public healthcare provider in the State of Qatar. The aim of this narrative review is to explore depression in cancer and discuss the need for screening for depression and to recommend suggestions and implications for future practice and research.
\end{abstract}

Key Words: Depression, Cancer, Screening, Prevalence

\section{INTRODUCTION}

Cancer is a major public health problem world-wide with a significant impact on the lives of many people. Studies have shown that cancer is experienced personally by one in four of the population, and it is estimated there will be 1,735,350 new cases worldwide by the year 2018. ${ }^{[1]}$ Like many other developing countries, the prevalence of cancer is increasing in the State of Qatar, partly because of a rapid transition in its socioeconomic status. ${ }^{[2,3]}$ The incidence of cancer among the population of the Middle East alone is predicted to rise by 50\%-70\% between 2009 and 2020. ${ }^{[2]}$ (p. 1077) A growing body of literature indicates that a cancer diagnosis may cause a significant psychological distress in up to $75 \%$ of cases. ${ }^{[4]}$ The most common psychological distresses in cancer patients include anxiety, depression, confusion, hostility, and anger. ${ }^{[5-7]}$ However, the rate of depression is estimated to be four times higher than that of the other psychological distresses. ${ }^{[8]}$ Based on prevalence studies, it is estimated that two in four to five cancer patients suffer from a major depressive episode after the time of diagnosis. ${ }^{[1,9]}$ Moreover, the prevalence of depression is higher in cancer patients than in the general population. ${ }^{[10]}$ This narrative review is built on a constructionist framework and to this end the purpose of this paper is to look toward the literature that seeks to highlight the need for screening for depression of those patients who are diagnosed with cancer.

\section{Methods}

Electronic databases including Cumulative Index of Nursing and Allied Health Literature (CINAHL), EvidenceBased Nursing, Evidence-Based Mental Health, MEDLINE, PubMed, and Cochrane Library were searched from 2000 until June 2015. Other databases including PsycINFO, Psy-

*Correspondence: Jessie Johnson; Email: Jessie.johnson1@ ucalgary.ca; Address: Faculty of Nursing, University of Calgary Qatar, Qatar. 
chology and Behavioral Sciences Collection, and Science Direct were also searched.

The initial search terms were kept broad by using wildcard characters, i.e., *, and MeSH terms to get a comprehensive literature search. These terms included "cancer" OR "neoplas*" OR "oncolog*”, AND "depression screening", AND "instrument" OR "scale" OR "survey" OR "assessment" OR "tool" OR "inventory". The search of the databases also included search for articles in Arab states of the Gulf region using the terms; "Cooperation Council for Arab States of the Gulf" OR "CCASG" OR "Gulf cooperation council" OR "Bahrain*” OR "Emirat*” OR "Kuwait*" OR "Oman*” OR "Qatar*" OR "Saudi Arabia*”. Other Arab countries included: "Egypt" OR "Iraq" OR "Jordan" OR "Lebanon" OR "Libya” OR "Palestine" OR "Syria" OR "Yemen".

The inclusion criteria were: (1) studies published in English from 2000 to the present (June/2015), (2) studies including adult cancer patients in a hospital setting, (3) studies developing and evaluating instruments used for screening depression in all types of cancer patients, (4) studies evaluating the psychometric properties of screening instruments, (5) studies comparing the psychometric properties of different screening instruments, (7) studies reporting the depression item separately while evaluating the effectiveness of screening instruments, and (8) available and accessible in online or printed formats.

Using a combination of these search terms resulted in 331 articles, which after examination of abstracts and inclusion/exclusion criteria 24 articles were selected. The reference lists of these articles identified two more articles that were deemed eligible. The final list included 26 articles for this narrative review.

\section{DEPRESSION}

Depression includes a range of feelings and emotions including normal sadness in response to loss of health, well-being, disability, and possible death. ${ }^{[1]}$ The 4 th edition of the Diagnostic and Statistical Manual (DSM-IV), published by the American Psychiatric Association (2000) ${ }^{[12]}$ has long been regarded as the gold standard of the diagnostic criteria for depression. The DSM-IV defines depression as the core symptoms of persistent low mood, and/or anhedonia (pervasive loss of pleasure/interest), lasting for two weeks or more. ${ }^{[12]}$ The DSM-IV describes two categories of depression, minor and major, mainly based on the severity of depressive symptoms. Major depression in characterized by having either low mood and/or loss of interest or pleasure (the core symptoms of depression) for at least two weeks. This is accompanied by at least four of the nine depressive symptoms, which include changes in sleep, changes in appetite, weight changes, agitation, fatigue, excessive guilt, difficulty thinking or concentration, psychomotor retardation, and recurrent thoughts of death or suicide ideation. ${ }^{[12]} \mathrm{Mi}-$ nor depression is characterized by having either low mood and/or anhedonia for two weeks or more, accompanied by at least two but not more than three of the depressive symptoms described by the DSM-IV.

\section{DEPRESSION AND CANCER}

Numerous studies have shown that depression is a substantial complication in cancer patients that may lead to a variety of psychological and physical (somatic) symptoms. These symptoms include fatigue, appetite disturbance or weight loss, sleep difficulties, difficulty with memory and concentration, an increase in pain perception, and a reduction in tolerance of side-effects of cancer treatment. ${ }^{[1,9]}$ Evidence suggests that these symptoms lead to a marked impairment in patients' functional capacity, social roles, quality of life, compliance with treatment, and may even have an effect on mortality. ${ }^{[11-13]}$ Studies have shown that depression in cancer patients is aggravated by fear of disability, fear of disfigurement, loss of control, uncertainty about the future, effects of the treatment, physical pain and financial worries. ${ }^{[14]}$ Moreover, depression may be influenced by the site, prognosis, and stage of cancer. ${ }^{[5,14]}$

Literature also indicates that almost half of cancer patients who have depression are not screened for depression, ${ }^{[15,16]}$ although the benefit of such screening has been well established. ${ }^{[17-20]}$ A report from the European Association for Palliative Care indicates that depression may remain unrecognized in more than $50 \%$ of cancer patients. ${ }^{[21]}$ According to the American Psychiatric Association (2000), despite the high levels of depression in cancer patients, the lack of screening and under-diagnosis of depression continue to be common. Hardman, Maguire, \& Crowther (2006) conducted a study of clinicians' ability to recognize depression in oncology patients. ${ }^{[2]}$ They found that physicians and nurses recognized only half of the depressed patients on a medical oncology service. Similarly, Jones and Doebbeling (2007) reported that depression is identified in less than one third of cancer patients, which is lower than the $50 \%$ recognition rate in the general population. ${ }^{[14]}$ Moreover, recent studies have shown that depression is an independent predictive factor for cancer-related mortality. ${ }^{[23,24]}$ Evidence indicates that unrecognized depression can be especially serious, causing unnecessary medical evaluation, longer hospital stays, disability, medical illness, and increased mortality due to suicide or accidental death. ${ }^{[16,25]}$

Depression in cancer patients is totally avoidable and treat- 
able once recognized. Torta and Ieraci (2013) state, "it is a serious clinical mistake to consider depression in oncological patients as an unavoidable pathology and consequently not responsive to drugs"[10] (p. 1132). A variety of pharmacological and psychosocial interventions have been developed specifically to treat depression in cancer patients. ${ }^{[19]}$ However, several factors play a part in the under-diagnosis and lack of recognition of depression among cancer patients. ${ }^{[15,16,19]}$ For example, symptoms like fatigue, weight loss, and concentration problems, which can result from depression, can be confused with the somatic (physical) symptoms of the underlying cancer itself. ${ }^{[25]}$ Furthermore, both physicians and cancer patients find it difficult to address emotional problems. ${ }^{[18,26]}$ Moreover, the frequent assumption by healthcare providers that all cancer patients are "understandably depressed" is another most common factor that plays a role in the under-diagnosis of depression in cancer patients. ${ }^{[15]}$ This may explain why the diagnosis of depression in cancer patients may easily be missed.

Evidence indicates that screening is one of the most efficacious, cost-effective, and feasible ways to recognize cancer patients who are at risk of depression. ${ }^{[14,26]}$ Screening, as defined by the UK National Screening Committee, is a "systematic application of a test or enquiry, to identify individuals at sufficient risk of a specific disorder to warrant further investigation or direct preventive action, amongst persons who have not sought medical attention on account of symptoms of that disorder"'[27] (p. 14).

\section{SCREENING FOR DEPRESSION}

A growing body of literature indicates the importance of screening cancer patients for depression. The National Comprehensive Cancer Network (NCCN) has recommended screening for depression in cancer patients as a standard of cancer care). ${ }^{[14]}$ A 2002 US National Institutes of Health (NIH) State-of-the-Science Conference Statement called for routine use of screening instrument to identify untreated and under-diagnosed depression in cancer patients. ${ }^{[28]}$ Moreover, the Institute of Medicine (IOM), the United States Preventive Services Task Forces (USPSTF), the American Society of Clinical Oncology (ASCO) and the UK National Institute for Clinical Excellence (NICE) have also recommended screening for psychological "distress" including depression, in cancer patients. ${ }^{[14,26,29]}$ The Pan-Canadian Practice Guidelines recommend that all cancer patients be screened for depression at their initial visit, at appropriate intervals, and as indicated clinically, especially with disease or treatment changes and transition to palliative care. ${ }^{[26]}$ Moreover, the guidelines recommend that screening should be done using valid and reliable instruments with established cut-offs that

Published by Sciedu Press are clinically meaningful. ${ }^{[26]}$

For this purpose, a variety of screening instruments have been developed over the past three decades to screen cancer patients for depression. Some of these instruments include: the Hospital Anxiety and Depression Scale (HADS), the Brief Case Find for Depression (BCD), the Hamilton Rating Scale for Depression (HRSD), the Beck Depression Inventory (BDI), the Center for Epidemiological Studies of Depression (CES-D), the Montgomery-Asberg Depression Rating Scale (MADRS), the Edmonton System Assessment System (ESAS), the Distress Thermometer (DT), and the Patient Health Questionnaire (PHQ). However, studies have shown that many of these screening instruments may not provide an accurate evaluation of depression in cancer patients because of the complex relationship between the physical symptoms of cancer itself and the symptoms of depression. ${ }^{[30,31]}$ Some studies have indicated that these instruments seem to measure physical symptom burden as well, especially when cancer patients suffer from clusters of symptoms such as fatigue/anorexia/cachexia, neuropsychology, debility, or pain. ${ }^{[14,32]}$ Thus, interpreting the results of screening instruments in these patients may be more challenging and, hence, a complete assessment is essential. Despite the increasing rate of cancer in the State of Qatar, to the best knowledge of this author, no published study has been conducted on the prevalence and screening of depression in cancer patients. Furthermore, discussion with oncologists, clinical nurse specialists, and staff nurses at the National Center for Cancer Care and Research (NCCCR) also revealed a lack of depression screening in cancer patients upon their assessment.

\section{Conclusion}

The findings of this review show that depression is one of the most common psychological distress in cancer patients. Yet often it is an unrecognized source of suffering among them. The known clinical implications of unrecognized depression highlight the importance of providing routine depression screening for cancer patients to help prevent adverse patient outcomes. Therefore, the findings of this review emphasize that oncology care providers must be able to identify cancer patients for possible depression, and should be familiar with the screening and diagnostic approaches that are available. For this reason, several depression screening instruments have been developed specifically for cancer patients. The use of these screening instruments are strongly recommended by several cancer care organizations including, the UK National Screening Committee, the National Comprehensive Cancer Network (NCCN), the US National Institutes of Health $(\mathrm{NIH})$, and the American Society of Clinical Oncology (ASCO). However, it is important for 
clinicians in oncology to understand that no screening instrument can completely replace a psychiatric clinical interview in diagnosing depression in cancer patients.

\section{RECOMMENDATIONS}

The findings of this narrative review suggest a number of recommendations as well as future research implications for health care providers. Currently, while there are no depression screening practices at the NCCCR, the recommendations from this study are relevant to both oncologists and nurses, as both routinely spend time with patients. Based on the evidence from this review, there is a strong need to implement a standardized, easy-to-use instrument to screen depression in cancer patients. However, one of the significant findings of this review is the contextual validation of the word "depression". The findings of this review indicate that the word "depression" may have different meanings and understandings in different cultures. ${ }^{[23,33]}$ Therefore, the multi-ethnic population in the State of Qatar may make it difficult to evaluate in populations where their first language is not English unless these screening tools are translated into Arabic.

Oncology nurses and educators play an important role in educating all clinicians regarding the need for routine depression screening in cancer patients, including depression screening protocol for oncology clinics. Moreover, nurses may also take the lead in educating patients that it is part of the normal routine for healthcare providers to ask patients about their mood and loss of interest. The recommendation to screen cancer patients for depression apply to other healthcare providers as well who routinely interact with cancer patients, such as social workers and radiation therapists.

This review also raises a number of implications for future research. First, a qualitative research study is needed to investigate the attitudes of healthcare providers, mainly oncologists and nurses, toward depression screening in cancer patients, to understand their depression screening behaviors and to help clarify the lack of depression screening practices among oncologists and nurses at NCCCR. Second, research is needed to explore how frequently and on what basis cancer patients are referred to psychosocial care at NCCCR since there are no depression screening practices. Third, welldesigned and executed randomized controlled trials (RCTs) are needed to investigate the benefits of depression screening in cancer patients to support recommendations for the incorporation of routine depression screening into standard cancer care. Such studies would make important contributions to nursing research, would help in understanding healthcare providers' depression screening attitudes, and would encourage the implementation of depression screening protocols at NCCCR.

\section{CONFlicts OF INTEREST Disclosure}

The authors declare that there is no conflict of interest.

\section{REFERENCES}

[1] American Cancer Society. 2012. Cancer facts and figures 2012. Available from: http://www. cancer.org/Research/CancerFa ctsFigures/CancerFactsFigures/index

[2] Bener A, Ayub H, Kakil R, et al. Patterns of cancer incidence among the population of Qatar: A worldwide comparative study. Asian Pacific Journal of Cancer Prevention. 2007; 8: 19-24.

[3] Brown R, Kerr K, Haoud A, et al. Tackling cancer burden in the Middle East: Qatar as an example. Lancet Oncology. 2012; 13: 501-508. https://doi.org/10.1016/S1470-2045(12) 70461-8

[4] Galway K, Black A, Cantwell M, et al. Psychosocial interventions to improve quality of life and emotional wellbeing for recently diagnosed cancer patients (Review). Cochrane Database of Systematic Reviews. 2010; 11: 1-68.

[5] Chochinov HM. Depression in cancer patients. The Lancet Oncology. 2001; 2: 499-505. https://doi.org/10.1016/S1470-2045(01 00456-9

[6] Curtis R, Groarke A, Sullivan F. Stress and self-efficacy predict psychological adjustment at diagnosis of prostate cancer. Scientific Reports. 2014; 4(4): 1-5.

[7] National Cancer Institute. Adjustment to Cancer: Anxiety and Distress-for health professionals. 2014. Available from: http://www. cancer.gov/about-cancer/coping/feelings/ anxiety-distress-hp-pdq\#section/_37

[8] Rudy R, House A. Psychosocial interventions in depressed cancer patients. Journal of Psychosocial Oncology. 2009; 29(5): 552-560.

[9] Ram S, Narayanasamy R, Barua A. Effectiveness of group psychoeducation on well-being and depression among breast cancer survivors of Melaka, Malaysia. Indian Journal of Palliative Care. 2013; 19(1): 34-39. PMid:23766593 https://doi.org/10.4103/0973 $-1075.110234$

[10] Torta RGV, Ieraci V. Pharmacological management of depression in patients with cancer: Practical considerations. Drugs. 2013; 73: 1131 1145. PMid:23839658 https://doi .org/10.1007/s40265-013 $-0090-7$

[11] Hosaka T, Aoki T. Depression among cancer patients. European Journal of Cancer Care. 2010; 19(6): 261-275.

[12] American Psychiatric Association. The Diagnostic and Statistical Manual IV (DSM-IV). Available from: http://www.psychiatry .org/practice/dsm

[13] Hopko DR, Bell JL, Armento M, et al. Cognitive-behavior therapy for depressed cancer patients in a medical care setting. Behavior Therapy. 2008; 39: 126-136. PMid:18502246 https ://doi .org/ $10.1016 / \mathrm{j}$. beth. 2007.05 .007 
[14] Jones D, Vichaya EG, Cleeland CS, et al. Screening for depressed mood in patients with cancer using the MD Anderson Symptom Inventory: Investigation of a practical approach for the oncologist. American Society of Clinical Oncology. 2014; 10(2): 95-102. PMid:24281151 https://doi.org/10.1200/JOP.2013.00111 2

[15] Pascoe S, Edelman S, Kidman A. Prevalence of psychological distress and use of support services by cancer patients at Sydney hospitals. Australian and New Zealand Journal of Psychiatry. 2000; 34: 785791. PMid:11037364 https://doi.org/10.1080/j.1440-161 $4.2000 .00817 . x$

[16] Lawrie I, Lloyd-Williams M, Taylor F. How do palliative medicine physicians assess and manage depression. Palliative Medicine. 2004; 18: 234-238. PMid:15198136 https ://doi.org/10.1191/0269 216304pm865oa

[17] Breitbart W. Identifying patients at risk for, and treatment of major psychiatric complications of cancer. Supportive Care in Cancer. 1995; 3: 45-60. https://doi.org/10.1007/BF00343921

[18] Newell SA, Sanson-Fisher RW, Savolainen NJ. Systematic review of psychological therapies for cancer patients: Overview and recommendations for future research. Journal of the National Cancer Institute. 2002; 94: 558-584. https://doi.org/10.1093/jnci /94.8.558

[19] Rodin G, Lloyd N, Katz M. The treatment of depression in cancer patients: A systematic review. Supportive Care in Cancer. 2007; 47: 702-712.

[20] Strong V, Water R, Hibberd C. Management of depression for people with cancer: A randomized controlled trial. Lancet. 2008; 372: 40-48. https://doi.org/10.1016/S0140-6736(08)60991-5

[21] Stiefel R, Die TM, Berney A, et al. Depression in palliative care: A pragmatic report from the Expert Working Group of the European Association for Palliative Care. Support Care Cancer. 2001; 9: 477-488. https://doi.org/10.1007/s005200100244

[22] Hardman A, Maguire P, Crowther D. The recognition of psychiatric morbidity on medical oncology ward. Cancer Practice. 2006; 21(3): 65-71.

[23] Lloyd-Williams M, Dennis M, Taylor F. Depression: An independent predictor of early death in patients with advanced cancer. Journal of Affective Disorders. 2009; 113: 127-132. PMid:18558439 https://doi.org/10.1016/j.jad.2008.04.002

[24] Satin JR, Linden W, Philips MJ. Depression as a predictor of disease progression and mortality in cancer patients: A meta-analysis. Can- cer. 2009; 115: 5349-5361. PMid:19753617 https ://doi.org/10 $.1002 /$ cncr. 24561

[25] Fawcett J. The morbidity and mortality of clinical depression. International Clinical Psychopharmacology. 2000; 8: 217-220. https: //doi.org/10.1097/00004850-199300840-00002

[26] Anderson WG, Alexander SC, Rodriquez KL. "What concerns me is"... Expression of emotion by advanced cancer patients during outpatient visits. Support Care Cancer. 2008; 16: 803-811. PMid:17960430 https://doi.org/10.1007/s00520-007-035 0-8

[27] UK National Screening Committee. First report of the national screening committee. 1998. Available from: http://aogm.org.mo/assets/Uploads/aogm/Guidelin es/NHS/National-Screening-Committee-UK-1998.pdf

[28] Patrick DL, Ferketich SL, Frame PS, et al. National Institutes of Health State-of-the Science conference statement: Symptom management in cancer, pain, depression, and fatigue. Journal of the National Cancer Institute. 2003; 95(15): 1110-1117. https: //doi.org/10.1093/jnci/djg014

[29] Meijer A, Roseman M, Milette K, et al. Depression screening and patient outcomes in cancer: A systematic review. Public Library of Science ONE. 2011; 6(11): 1-13.

[30] Al-Adawi S, Dorvlo ASS, Al-Naamani A, et al. The ineffectiveness of the Hospital Anxiety and Depression Scale for diagnosis in an Omani traumatic brain injured population. Brain Injury. 2007; 21(4): 385-393. PMid:17487636 https : //doi .org/10.1080/02 699050701311059

[31] Lazenby M, Dixon J, Bai M, et al. Comparing the distress thermometer (DT) with the patient health questionnaire (PHQ)-2 for screening for possible cases of depression among patients newly diagnosed with advanced cancer. Palliative and Supportive Care. 2014; 12: 63-68. PMid:24468423 https://doi.org/10.1017/S1478951513000 394

[32] Tu C, Hsu M, Chi S, et al. Routine depression screening and diagnosing strategy for cancer inpatients. Psycho-Oncology. 2014; 23: 10571067. PMid:24798464 https ://doi .org/10.1002/pon. 3547

[33] Warmenhoven F, Rijswijk EV, Engels Y, et al. The Beck Depression Inventory (BDI-II) and a single screening question as screening tools for depressive disorder in Dutch advanced cancer patients. Support Care Cancer. 2012; 20: 319-324. PMid:21243377 https://doi.org/10.1007/s00520-010-1082-8 